\section{Hypercalcemia and a "no observed adverse effect level" intake of vitamin D}

There are many reasons why the diagnosis of vitamin D toxicity in the recent case report by Auguste and colleagues ${ }^{1}$ was probably wrong and the result of a red herring. The patient had been taking 8000-10000 IU of vitamin D daily for 2.5 years, during which time serum creatinine levels were not an issue. When the patient's serum 25-hydroxyvitamin D was first measured, it was $241 \mathrm{nmol} / \mathrm{L}$, consistent with the patient's reported long-term vitamin D intake but almost double the top of the reference range for people not taking a supplement. ${ }^{2}$ However, the serum 1,25-dihydroxyvitamin D level was exceptionally high, along with serum calcium and creatinine levels.

The Institute of Medicine specifies $10000 \mathrm{IU} / \mathrm{d}$ as the "no observed adverse effect level" - an intake that is not advisable, but not considered objectively harmful either. ${ }^{2}$ Doses of vitamin D higher than $10000 \mathrm{IU} / \mathrm{d}$ have been used in clinical trials that achieved higher 25-hydroxyvitamin D values and for longer duration, yet there was not one case of hypercalcemia, and certainly no kidney damage reported from among the hundreds of those study participants. ${ }^{3-9}$

The case report described by Auguste and colleagues is not consistent with any previous clinical experience with vitamin $D$ intake. What is unusual is the high serum 1,25-dihydroxyvitamin D level, because even in the most extreme cases of vitamin D toxicity, with 25-hydroxyvitamin D exceeding $2000 \mathrm{nmol} / \mathrm{L}$, the total serum 1,25-dihydroxyvitamin D was only modestly increased. ${ }^{10}$ The primary cause of the renal impairment in the case report of Auguste and colleagues was something beyond the vitamin $D$ intake: either a tumour or sarcoidosis.

Auguste and colleagues speculate that the patient might have been unusually susceptible to vitamin $D$ because of a mutation in the CYP24A1 gene that encodes for the breakdown enzyme of 25-hydroxyvitamin D and 1,25- dihydroxyvitamin D. ${ }^{1}$ But if that were the case, the condition would not have required 2.5 years of vitamin $D$ supplementation to manifest itself, and it could not have been resolved within a few months. Since several genetic defects can impair the CYP24A1 enzyme, the diagnosis is suitably screened for with a biochemistry laboratory test, the ratio of 25-hydroxyvitamin D to 24,25dihydroxyvitamin D concentrations. ${ }^{11}$ That test was conducted for my research group at the same hospital laboratory that Auguste and colleagues used for their own 25-hydroxyvitamin D results. ${ }^{12}$ It is not likely that a rare CYP24A1 defect is pertinent to this case report, because of the duration of vitamin D intake, the elevated 1,25dihydroxyvitamin $\mathrm{D}$ and the recent onset of symptoms.

The high serum 1,25-dihydroxyvitamin D levels, hypercalcemia-related renal impairment and recent onset are entirely consistent with published case reports on sarcoidosis. ${ }^{13,14}$ The only difference was that, in those cases, serum 25-hydroxyvitamin D levels were normal. The high 1,25-dihydroxyvitamin D in the case report of Auguste and colleagues ${ }^{1}$ is not a sign of vitamin $D$ toxicity and is likely a consequence of sarcoidosis. ${ }^{13-15}$ The renal biopsy used by Auguste and colleagues does not rule out sarcoidosis. ${ }^{16}$ The hydroxychloroquine they used to treat the patient is not a conventional treatment for vitamin D toxicity, but it is a first-line treatment for sarcoidosis. ${ }^{13-15}$ Although the case reported by Auguste and colleagues was probably not a primary disease of vitamin D toxicity, it is important to limit sources of vitamin D in patients with sarcoidosis.

The lesson here is that it is common for patients to take dietary supplements in amounts that may raise test values beyond the laboratory's reference range. But an abnormally high laboratory value does not in itself justify a diagnosis of toxicity. The consequence of accepting the false clue of a high vitamin D level was that it curtailed further effort to establish the true cause of the problem.

\section{Reinhold Vieth PhD}

Professor emeritus, Departments of Laboratory Medicine and Pathobiology, and Nutritional Sciences, University of Toronto, Toronto, Ont.

Cite as: CMAJ 2019 July 8;191:E768. doi: 10.1503/cmaj.72512

\section{References}

1. Auguste BL, Avila-Casado C, Bargman JM. Use of vitamin $D$ drops leading to kidney failure in a 54-year-old man. CMAJ 2019;191:E390-4.

2. Institute of Medicine. Dietary reference intakes for calcium and vitamin D. Washington (DC): The National Academies Press; 2011.

3. Vieth R. Vitamin D supplementation, 25hydroxyvitamin $\mathrm{D}$ concentrations, and safety. $\mathrm{Am} \mathrm{J}$ Clin Nutr 1999;69:842-56.

4. Hathcock JN, Shao A, Vieth R, et al. Risk assessment for vitamin D. Am J Clin Nutr 2007;85:6-18.

5. Hasling C, Nielsen HE, Melsen F, et al. Safety of osteoporosis treatment with sodium fluoride, calcium phosphate and vitamin D. Miner Electrolyte Metab 1987;13:96-103.

6. Wagner D, Trudel D, Van der Kwast T, et al. Randomized clinical trial of vitamin $D_{3}$ doses on prostatic vitamin D metabolite levels and ki67 labeling in prostate cancer patients. J Clin Endocrinol Metab 2013;98:1498-507.

7. Kimball SM, Ursell MR, O'Connor P, et al. Safety of vitamin $\mathrm{D}_{3}$ in adults with multiple sclerosis. $A m$ J Clin Nutr 2007;86:645-51.

8. Smolders J, Peelen E, Thewissen M, et al. Safety and $T$ cell modulating effects of high dose vitamin $\mathrm{D}_{3}$ supplementation in multiple sclerosis. PLoS One 2010;5:e15235.

9. Burton JM, Kimball S, Vieth R, et al. A phase I/II dose-escalation trial of vitamin $D_{3}$ and calcium in multiple sclerosis. Neurology 2010;74:1852-9.

10. Vieth R, Pinto TR, Reen BS, et al. Vitamin D poisoning by table sugar. Lancet 2002;359:672.

11. Molin A, Baudoin R, Kaufmann M, et al. CYP24A1 mutations in a cohort of hypercalcemic patients: evidence for a recessive trait. J Clin Endocrinol Metab 2015;100:E1343-52.

12. Wagner D, Hanwell HE, Schnabl K, et al. The ratio of serum 24,25-dihydroxyvitamin $D_{3}$ to 25 -hydroxyvita$\min D_{3}$ is predictive of 25 -hydroxyvitamin $D_{3}$ response to vitamin $D_{3}$ supplementation. J Steroid Biochem Mol Biol 2011;126:72-7.

13. Hanna RM, Kaldas M, Arman F, et al. Hypercalcemiainduced acute kidney injury in a Caucasian female due to radiographically silent systemic sarcoidosis. Clin Nephrol Case Stud 2018;6:21-6.

14. Ueda S, Murakami T, Ogino H, et al. Systemic sarcoidosis presenting with renal involvement caused by various sarcoidosis-associated pathophysiological conditions. Intern Med 2019;58:679-84.

15. Sharma OP. Effectiveness of chloroquine and hydroxychloroquine in treating selected patients with sarcoidosis with neurological involvement. Arch Neurol 1998;55:1248-54.

16. Prasse A. The diagnosis, differential diagnosis, and treatment of sarcoidosis. Dtsch Arztebl Int 2016;113:565-74.

Competing interests: Reinhold Vieth receives patent royalties from Ddrops (vitamin D supplement). 